\title{
Hypogonadal Men with Type 2 Diabetes Benefit from Testosterone Treatment: 10-Year Real-Life Data from a Registry Study
}

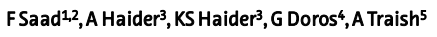 \\ ${ }^{1}$ Medical Affairs Andrology, Bayer AG, Berlin, Germany \\ 2 Gulf Medical University School of Medicine, Ajman, UA \\ ${ }^{3}$ Private Urology Practice, Bremerhaven, Germany \\ "Department of Epidemiology and Statistics, Boston University School of Public Health, Boston, MA, USA \\ Department of Biochemistry and Department of Urology, Boston University School of Medicine, Boston, MA, USA
}

\begin{abstract}
Background
Prevalence of hypogonadism in men with type 2 diabetes mellitus (T2DM) can be as high as $50 \%$.
Tim from an ongoing registry study in a urological setting.

Methods

A registry of hypogonadal men was established in $2004 \mathrm{in}$ a single urologist's office. All men had testosterone $\leq 12.1 \mathrm{nmol} / \mathrm{L}$. In a total 776 patients, $286(36.9 \%)$ had type 2 diabetes which had been diagnosed and is being treated elsewhere (family

physician, diabetologist).
Of these, 133 received TU $1000 \mathrm{mg} / 12$ weeks (T-group) following an initial 6-week interval. 153 had opted against TTh and served as controls (CTRL). Measurements were performed 1-4 times a year for up to 11 years and included, anthropometric parameters, glycaemic control and concomitant diabetes medication. 10 -year data are reported. Mean changes over time
between groups were compared by mixed effects model for repeated measures with random effect for intercept and fixed between groups were compared by mixed effects model tor repeated measures with random effect for intercept and fixed
effects for time, group and their interaction and adjusted for age, weight, waist circumference, fasting glucose, blood effects for time, group anc their interaction and adjusted for age, weight, waist circumference, fasting glucose, blood
pressureand lipids to account for baseline differences between groups. Mean follow-up was 6.9 years in the T-group and 7.3 years in CTRL, median follow-up 7 years in the T-group and 8 years in CTRL.
\end{abstract}

\section{Results
Mean age at baseline was $61.8 \pm 5.4$ years in the T-group and $64.2 \pm 4.6$ years in CTRL.}

Mean $\mathrm{HbA}_{1}$ progressively decreased from $8.8 \pm 0.9$ to $5.9 \pm 0.3 \%$ after 10 years in the $\mathrm{T}$-group $(p<0.0001)$. The decrease was statistically significant vs, previous year for the first 8 years, then continued without reaching statistical significance. In

CTRL, mean $\mathrm{Hb} A_{11}$ increased from $7.7 \pm 0.6$ to $9.5 \pm 0.9 \%$ ( $p<0.0001$ ). The estimated adjusted difference between groups was $-4.6 \%(p<0.0001)$.

Fasting glucose decreased from $7.6 \pm 1.1$ to $5.3 \pm 0.1 \mathrm{mmol} / \mathrm{L}(p<0.0001)$ in the $T$-group. The decrease was statistically significant vs. previous year for the first 3 years. In CTRL, fasting glucose increased from $6.2 \pm 0.6$ to $6.9 \pm 1.0 \mathrm{mmo} / / \mathrm{L}(\mathrm{p}$ $<0.0001)$. The estimated adjusted difference between groups was $-2.2 \mathrm{mmol} / \mathrm{L}(\mathrm{p}<0.0001)$.

HOMA-IR decreased from $10.2 \pm 2.0$ to $3.6 \pm 1.2$ after 10 years $(\mathfrak{p}<0.0001)$. The decrease was statistically significant vs.
Previous year for the first 5 years. In CTRL, HOMA-IR increased from $7.4 \pm 1.4$ to 11.4 ( $p<0.0001)$. The estimated adjusted diffe rence between groups was $-9.4 \mathrm{mmo} / \mathrm{L}(\mathrm{p}<0.0001)$.

Fasting insulin decreased from $29.6 \pm 4.2$ to $15 \pm 4.8 \mu \mathrm{U} / \mathrm{mL}(\mathrm{p}<0.0001)$ with statistical significance vs. previous year for the first 7 years. In CTRL, fasting insulin increased from $26.5 \pm 2.6$ to $37 \mu \mathrm{U} / \mathrm{mL}(\mathrm{p}<0.0001)$. The estimated adjusted difference between groups was $-23.1 \mu \mathrm{U} / \mathrm{mL}(\mathrm{p}<0.0001)$.

At baseline, 54 patients in the T-group recelved insulin at a mean dose of $32.4 \pm 12.1$ units/day.Mean dose requirement declined to $20.6 \pm 11.3$ units $(p<0.0001)$ with statistical significance vs. previous year for the first 7 years. In CTRL, 55 patient received insulin at a mean dose of $29.4 \pm 5$ units/day. Mean dose requirement increased to $39.5 \pm 7.2$ units $(p<0.0001)$. The estimated adjusted difference between groups was -25.7 units/day $(\mathrm{p}<0.0001)$.

In the T-group, $106(80 \%)$ achieved $\mathrm{HbA}_{1 c}<6.5 \%$, and $116(87 \%)$ achieved $\mathrm{HbA}_{1 c}<7 \%$ at last measurement. In CTRL, no patien achieved either $\mathrm{HbA}_{1 c}<6.5 \%$ or $\mathrm{HbA}_{1 c}<7.0 \%$. All but 1 man had an increase in $\mathrm{HbA}_{1 c}$

In the T-group, 4 patients ( $3 \%$ ) died of non-cardiovascular events. There were no non-fatal major adverse cardiovascular events (MACE). In CTRL, 24 patients (15.7\%) died. 50 non-fatal MACE occurred (26 myocardial infarctions, 24 strokes). Since all injectio
treatment.

\section{Discussion}

imitations: The study was notdesigned to investigate effects of testosteroneon type 2 diabes. Also, patientswerenot randomised. In a regular medical office, neither randomisation nor placebo-control were an option. After receiving inform tion about testosterone treatment, to undergo testosterone therapy was the patients' own choice. There was no information about adherence to antidiabetic medications. The untreated control patients were approximately 2.5 years older at baseline. However, their baseline diabetes parameters were more favourable than they were in the testosterone-treated patients. Strengths: The long duration of follow-up the excellent medication adherence to testosterone, and close interdisciplinary collaboration with the diabetologists.

Conclusion: long-term treatment with TU in hypogonadal men with T2DM improved glycaemic control compared to untreated controls. In patients in the T-group, insulin dose could be substantially reduced. Correcting hypogonadism in men with T2DM sustainably supports standard diabetes treatment.
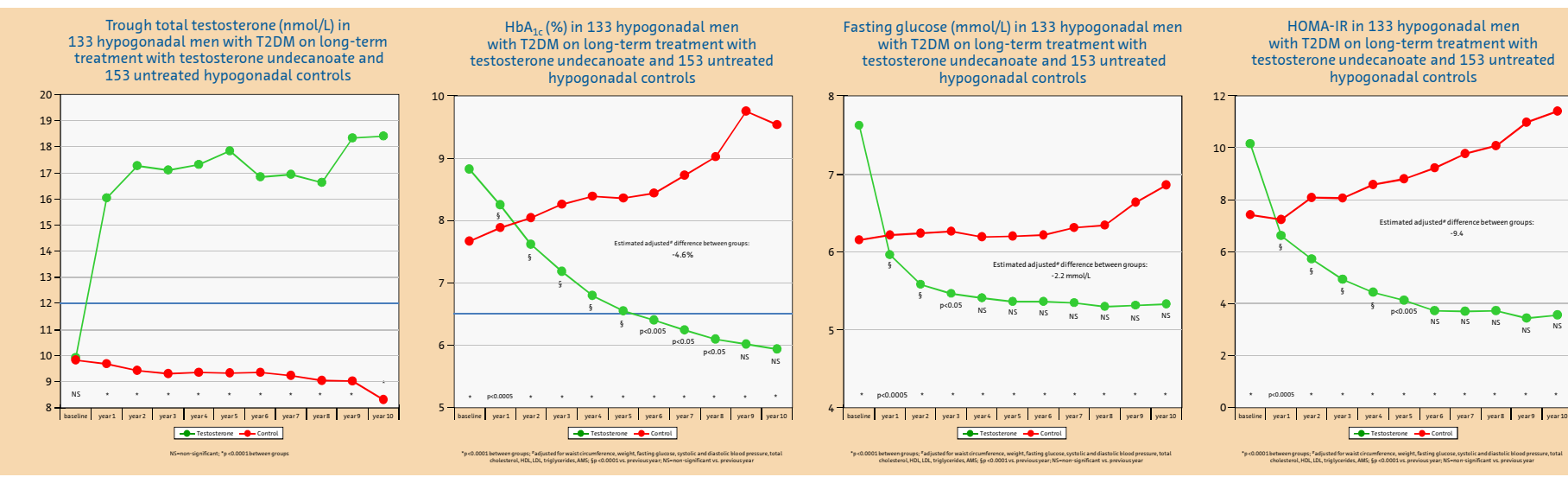

Fasting insulin $(\mu \mathrm{U} / \mathrm{mL})$ in 133 hypogonadal men testosterone undecanoate and 153 untreated hypogonadal controls

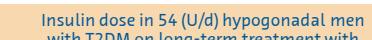
erm treatment with hypogonadal controls
hypons
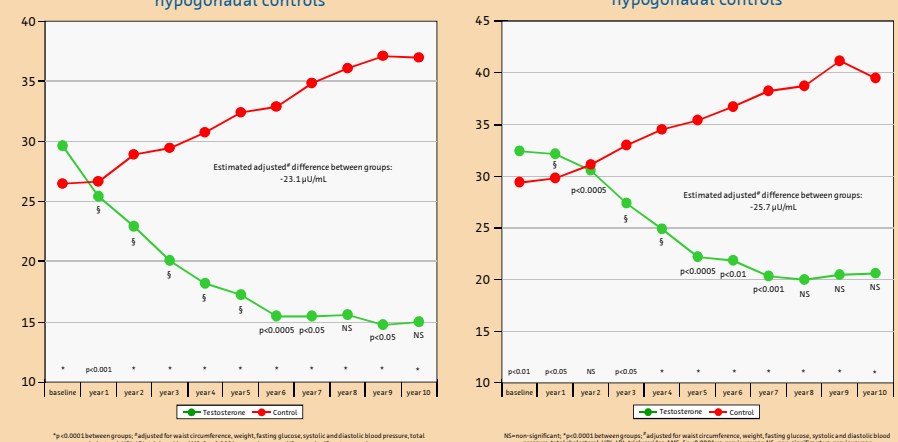

Proportion of patients achieving $\mathrm{HbA}_{1 \mathrm{c}}$ targets of $6.5 \%$ and $7.0 \%$, respectively

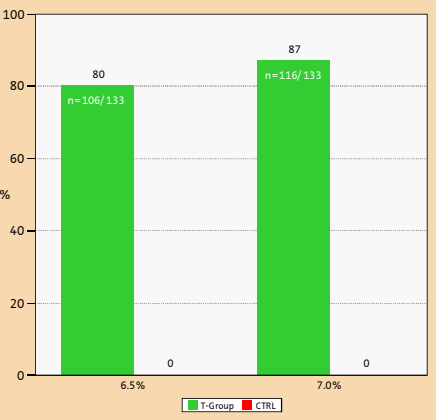

Proportion of deaths, Mis and strokes T-Group and CTRL

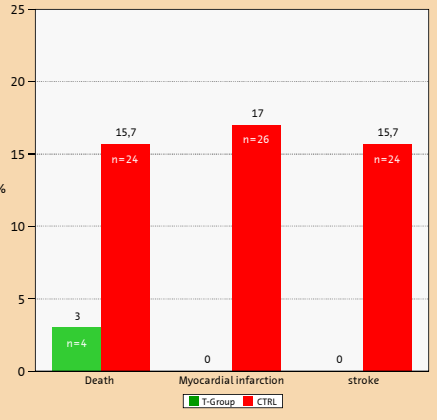

